\title{
Incidence, type of atrial fibrillation and risk factors for stroke: a population-based cohort study
}

\section{Cecilia Johansson \\ Erik Dahlqvist \\ Jonas Andersson \\ Jan-Håkan Jansson \\ Lars Johansson}

Department of Public Health and Clinical Medicine, Skellefteå Research Unit, Umeå University, Umeå,

Västerbotten, Sweden

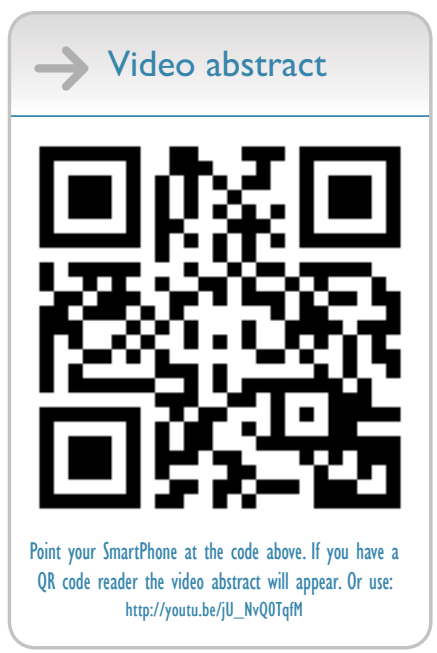

Correspondence: Cecilia Johansson Department of Public Health and Clinical Medicine, Skellefteå Research Unit, Skellefteå County Hospital, SE-93। 86 Skellefteå, Sweden

Tel +46 9 I077 I000

Fax $+4691077 \quad 1657$

Email cecjoh@gmail.com
This article was published in the following Dove Press journal:

Clinical Epidemiology

24 January 2017

Number of times this article has been viewed

Purpose: The aims of this study were to estimate the incidence of atrial fibrillation and atrial flutter (AF), to assess the presence of provoking factors and risk factors for stroke and systemic embolism, and to determine the type of AF in patients with first-diagnosed AF.

Patients and methods: This cohort study was performed in northern Sweden between January 1, 2011 and December 31, 2012. Diagnosis registries were searched for the International Classification of Diseases-10 code for AF (I48) to identify cases of incident AF. All AF diagnoses were electrocardiogram-verified. Data pertaining to provoking factors, type of AF and presence of risk factors for stroke and systemic embolism according to the $\mathrm{CHA}_{2} \mathrm{DS}_{2}$-VASc score were obtained from medical records.

Results: The incidence of AF in the entire population was 4.0 per 1,000 person-years. The incidence was 27.5 per 1,000 person-years in patients aged $\geq 80$ years. A total of $21 \%$ of all patients had a provoking factor in association with the first-diagnosed episode of AF. The $\mathrm{CHA}_{2} \mathrm{DS}_{2}$-VASc score was 2 or higher in $81 \%$ of the patients. Permanent AF was the most common type of AF (29\%).

Conclusion: There was a considerable increase in the incidence of AF with age, and a provoking factor was found in one-fifth. The most common type of AF was permanent AF. Four in five patients had a $\mathrm{CHA}_{2} \mathrm{DS}_{2}$-VASc score of 2 or more.

Keywords: atrial fibrillation, atrial flutter, incidence, risk factors for stroke

\section{Introduction}

Recent studies have reported that the prevalence rate of atrial fibrillation or atrial flutter (AF) is $2 \%-3 \% .{ }^{1-3}$ Only a few studies have investigated the incidence of AF, and the results have been inconclusive. The annual incidence of AF in subjects of all ages was 4.1 per 1,000 person-years in a German study and 0.9 per 1,000 person-years in a Scottish study. ${ }^{2,4}$ It is not uncommon for a provoking factor, such as surgery or infection, to be present when AF is first diagnosed. The Framingham study showed that $31 \%$ of the patients diagnosed with AF had a provoking factor at the time of their diagnosis of $\mathrm{AF}^{5}$

AF is a progressive disease, and in clinical practice it is classified as follows: firstdiagnosed $\mathrm{AF}$, paroxysmal $\mathrm{AF}$, persistent $\mathrm{AF}$ or permanent $\mathrm{AF}^{6,7}$ In a recent study of patients with newly diagnosed AF, 29.7\% had unclassified AF, 27.5\% had paroxysmal AF, $17.9 \%$ had persistent $\mathrm{AF}$ and $24.9 \%$ had permanent $\mathrm{AF}$ when they were classified up to six weeks after the first-detected episode. ${ }^{8}$ Notably, the prevalence of cardiovascular risk factors and other comorbidities varies between patients with the different types of AF. The proportion of patients with previous stroke, coronary artery disease, hypertension, 
heart failure, and diabetes mellitus is higher in patients with permanent AF compared to those with first-detected AF, paroxysmal AF and persistent AF. ${ }^{9}$ One study found that the median $\mathrm{CHA}_{2} \mathrm{DS}_{2}$-VASc score was 4.0 in patients with longstanding, persistent or permanent AF compared to that (3.0) in patients with first-detected AF, paroxysmal or persistent AF. ${ }^{10}$ The incidence of a cardiovascular disease such as ischemic heart disease, ischemic stroke or peripheral arterial disease, or hypertension or diabetes mellitus, was higher in patients with incident AF than in the general population. ${ }^{11}$

The distribution of the types of AF, the proportion of provoking factors associated with $\mathrm{AF}$ and the risk factors for stroke and systemic embolism depend on the population studied. Patients with AF who were recruited from hospital populations or who were referred to a cardiologist for assessment are not representative for the general AF population. In a previous study, we estimated the prevalence of AF in the total population within a well-defined geographical area in northern Sweden. ${ }^{1}$ The study reported here included patients with incident AF in this unselected "real-life" population, in order to estimate the incidence of AF and to characterize patients with first-diagnosed AF.

The aims of this study were to estimate the incidence of AF, to assess the presence of provoking factors and risk factors for stroke and systemic embolism, and to determine the type of AF in patients with first-diagnosed AF.

\section{Materials and methods}

\section{Setting and case finding}

This cohort study was performed in northern Sweden between January 1, 2011 and December 31, 2012. All patients in the study area with a first-diagnosed episode of AF during this time period were identified and included in the study. The patient characteristics were recorded from the first-diagnosed episode of AF up to six months after the diagnosis of AF to enable a better classification of the type of AF and risk factors for stroke. Patients were followed until the death or migration from the study area, or for maximum six months. The patient inclusion process is shown in Figure 1.

The study was performed in two Swedish municipalities, Skellefteå and Norsjö, which have a combined total of 75,779 inhabitants. Skellefteå County Hospital is the only hospital in the catchment area. Patients requiring specialized care, such as cardiac surgery, are referred to the nearby University Hospital and then referred back to the Skellefteå County Hospital for post-procedure care and follow-up. We used the National Patient Register (NPR) for Skellefteå County Hospital and a local diagnosis register of all primary healthcare providers in the area to identify the possible cases of AF. These registers show the International Classification of Diseases-10 (ICD-10) diagnosis codes from all inpatient care and outpatient visits in Sweden. ${ }^{12}$ Patients who received a primary or secondary diagnosis of AF (ICD-10 code I48)

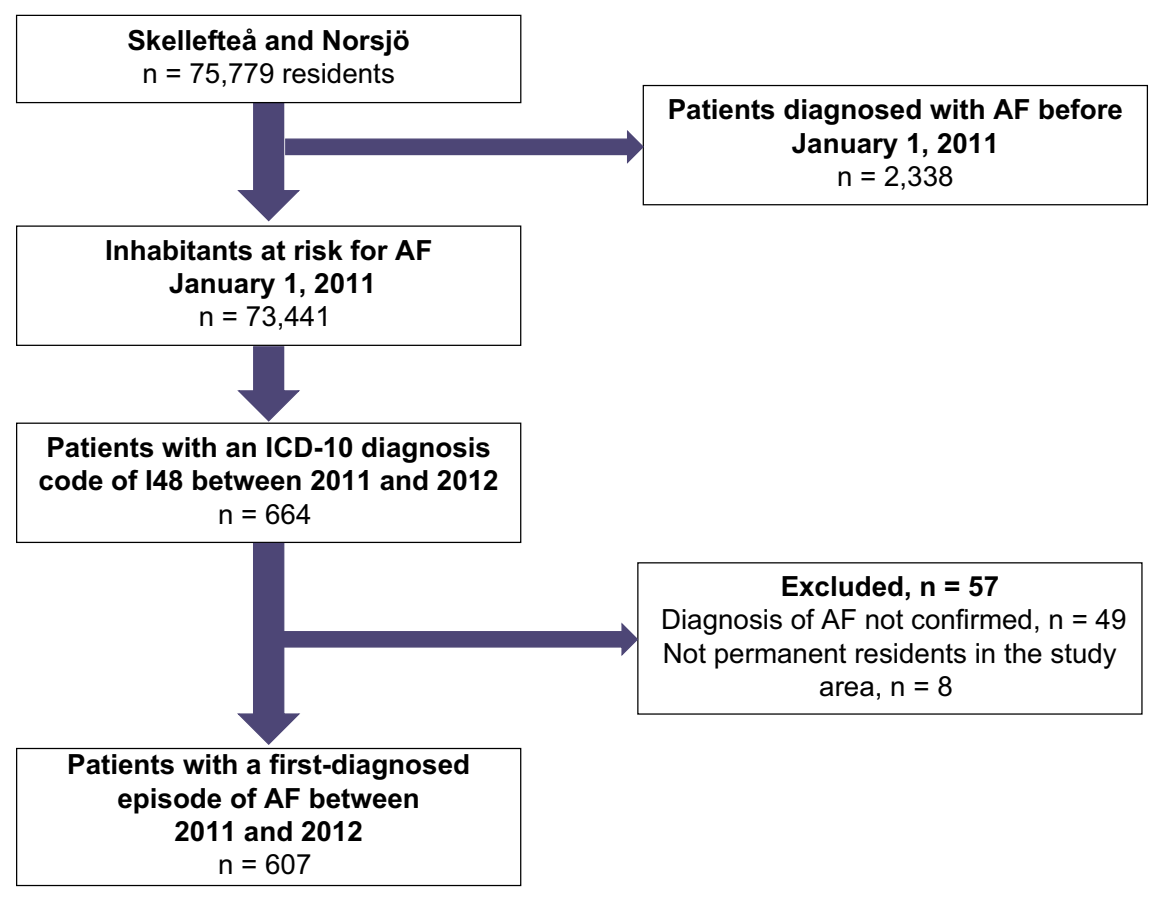

Figure I Flow chart showing the patients with atrial fibrillation or atrial flutter (AF) who were included in this study. Abbreviation: ICD-10, the International Classification of Diseases- 10. 
from January 1, 2011 to December 31, 2012 were considered to be the possible cases of first-diagnosed AF. Patients with a diagnosis of AF or an electrocardiogram (ECG) showing AF before January 1, $2011(n=2,274)$ were included in a previous study and were excluded from this one. ${ }^{13} \mathrm{We}$ also identified 64 additional patients with a diagnosis of AF before January 1, 2011, who were not included in the previous study. These additional 64 patients were excluded from the present study population; they had received their first diagnosis of AF outside the study region before January 1, 2011, mainly at the nearby University Hospital. All medical records and ECGs of possible cases of first-diagnosed AF were then individually validated by one of the authors. Patients with an unconfirmed diagnosis of AF $(n=49)$ and those who were not permanent residents in the study area $(n=8)$ were excluded from the study.

\section{Patient characteristics}

Medical data were extracted from the medical records of all primary healthcare centers in the area and from the medical records of all departments at Skellefteå County Hospital (excluding the departments of Gynecology, Psychiatry, Otorhinolaryngology and Ophtamology). Specifically, we collected data on the presence of a provoking factor in association with the diagnosis of AF. The type of AF and presence of risk factors for stroke and systemic embolism according to the $\mathrm{CHA}_{2} \mathrm{DS}_{2}-\mathrm{VASc}$ risk score (congestive heart failure, hypertension, age $\geq 75$ years [scored as two points], diabetes mellitus, previous stroke/transient ischemic attack [TIA]/arterial embolism [scored as two points], vascular disease, age 65-74 years and female sex) were collected up to six months after the first-diagnosed episode of AF. Date and the main cause of death were also registered from the death certificate. The detailed definitions of the risk factors for stroke and systemic embolism and the provoking factors for AF (eg, myocardial infarction, acute infection, surgery) are listed in Table S1.

\section{Classification of AF}

$\mathrm{AF}$ is, in this study, defined as atrial fibrillation or atrial flutter as they often coexist. ${ }^{14}$ The types of AF were classified as follows: 1) Single episode of AF: This was defined as one non-recurrent, ECG-documented, episode of AF that selfterminated within seven days of onset. There was no patient history indicating further episodes of $\mathrm{AF}$, and all subsequent ECG screenings showed sinus rhythm. 2) Paroxysmal AF: This was defined as recurrent, ECG-documented, episodes of AF or a single ECG-documented episode of AF plus a patient history, indicating further episodes of AF. All episodes of AF were self-terminating within seven days of onset. 3) Persistent AF: This was defined as AF for which cardioversion was performed or planned, or AF lasting more than seven days that did not fulfill the criteria for permanent AF. 4) Permanent AF: This was defined as AF at the last ECG plus acceptance of the presence of the arrhythmia by the supervising physician.

\section{Statistical analysis}

Means with standard deviations, numbers and proportions were calculated for baseline data. The incidence was calculated as the number of patients with first-diagnosed AF divided by the total population at risk for first-diagnosed AF (which equaled the total population in the study area, except for people who were already diagnosed with AF at the start of the study). Univariate and multivariate Cox proportional hazards regression analyses were used to investigate associations, which were presented as hazard ratios (HRs) and 95\% confidence intervals (CIs), between the presence of provoking factors for AF and mortality, adjusted for age and sex. SPSS version 22.0 was used for statistical calculations.

\section{Ethics}

This study was approved by the Regional Ethics Review Board of Umeå University. No patient consent to review their medical records was required by the Regional Ethics Review Board at Umeå University.

\section{Results \\ Incidence of AF}

We identified 607 patients with a first-diagnosed episode of AF in 2011 and 2012. The mean age of these patients was 73.5 years, and $42.3 \%$ were women (see Table 1). The

Table I Baseline table showing characteristics of 607 patients with first-diagnosed atrial fibrillation or atrial flutter (AF)

\begin{tabular}{ll}
\hline Age at diagnosis, years (mean) & $73.5 \pm 12.2$ \\
Female sex & $257(42.3)$ \\
Congestive heart failure & $110(18.1)$ \\
Hypertension & $380(62.6)$ \\
Diabetes mellitus & $10 I(16.6)$ \\
Previous stroke, TIA or arterial embolism & $113(18.6)$ \\
Vascular disease & $126(20.8)$ \\
$\mathrm{CHA}_{2} \mathrm{DS}_{2}$-VASc, mean & $3.3 \pm 1.9$ \\
$\mathrm{CHA}_{2} \mathrm{DS}_{2}$-VASc 0 points & $42(6.9)$ \\
$\mathrm{CHA}_{2} \mathrm{DS}_{2}$-VASc I point & $75(12.4)$ \\
$\mathrm{CHA}_{2} \mathrm{DS}_{2}$-VASc $\geq 2$ points & $490(80.7)$ \\
\hline
\end{tabular}

Notes: aPrevious myocardial infarction, objectively verified peripheral arterial disease, complex aortic plaque or previous surgery for an arterial aneurysm. $\mathrm{CHA}_{2} \mathrm{DS}_{2}$-VASc score for $\mathrm{AF}$ stroke risk: congestive heart failure, hypertension, age $\geq 75$ years (scored as 2 points), diabetes mellitus, previous stroke/TIA/arterial embolism (scored as 2 points), vascular disease, age 65-74 years and female sex. Data are reported as $\mathrm{n}(\%)$ or as mean \pm standard deviation.

Abbreviation: TIA, transient ischemic attack. 
incidence of AF in the total population was 4.0 cases per 1,000 person-years. The incidence increased with age and peaked in the age group 85-99 years for both men (44.5 per 1,000 person-years) and women (28.3 per 1,000 person-years; see Figure 2 and Table S2).

The reason for ECG monitoring leading to the first diagnosis of AF was suspected arrhythmia in 199 patients (32.8\%), whereof 127 patients had palpitations, 17 patients had syncope and 55 patients were asymptomatic but had irregular pulse at clinical examination. In 213 patients (35.1\%), ECG recordings were collected due to suspected ischemic heart disease (eg, symptoms of chest pain or dyspnea). AF was found due to screening in the context of stroke or TIA investigation in 35 patients $(5.8 \%)$. In 56 patients (9.2\%), AF was diagnosed by continuous ECG monitoring for other reasons. Finally, AF was found on routine examination (eg. preoperative check or a health examination in 104 patients $(17.1 \%)$.

AF was first diagnosed at primary healthcare in 156 patients $(25.7 \%)$ and at hospital inpatient and outpatient clinics (including emergency department and intensive care units) in 451 patients (74.3\%). When the first episode of AF was diagnosed, 82 patients $(13.5 \%)$ were primarily hospitalized due to AF.

\section{The presence of provoking factors}

A provoking factor was present in 125 patients $(20.6 \%)$. These include cardiac surgery (46 patients), acute infection
(24 patients), non-cardiac surgery (21 patients), myocardial infarction (16 patients), anemia (6 patients), thyrotoxicosis or overdosage of levothyroxine (4 patients), dehydration ( 2 patients), acute abdominal pain ( 2 patients), high tumor burden ( 2 patients), aortic aneurysm (1 patient) and status epilepticus ( 1 patient). Provoking factors were most common in patients with a single episode of AF and least common in those with permanent AF. Patients with a provoking factor present at the first diagnosis of AF had a mean age of $72.5 \pm 11.7$ years, and those with no provoking factor had a mean age of $73.8 \pm 12.3$ years. Patients who had a cardiac surgery as a provoking factor associated with their first diagnosis of AF were younger and more often men than those with no provoking factor (Table 2).

\section{Risk factors for stroke or systemic embolism}

The most common risk factors for stroke or systemic embolism were age ( $\geq 65$ years, 474 patients, $78.1 \%$ ) and hypertension (380 patients, $62.6 \%$ ). The mean $\mathrm{CHA}_{2} \mathrm{DS}_{2}$-VASc score was 3.3 , and $80.7 \%$ had a score of 2 or more. The risk factors for stroke are shown in Table 1. Among the 490 patients with a $\mathrm{CHA}_{2} \mathrm{DS}_{2}$-VASc score of 2 or more, $66.3 \%$ were treated with oral anticoagulants, and among the 75 patients with a $\mathrm{CHA}_{2} \mathrm{DS}_{2}$-VASc score of $1,62.7 \%$ were treated with oral anticoagulants. Among the 42 patients with a $\mathrm{CHA}_{2} \mathrm{DS}_{2}$-VASc score of $0,16(38.1 \%)$ were treated with oral anticoagulants (of whom 13 were scheduled for cardioversion).

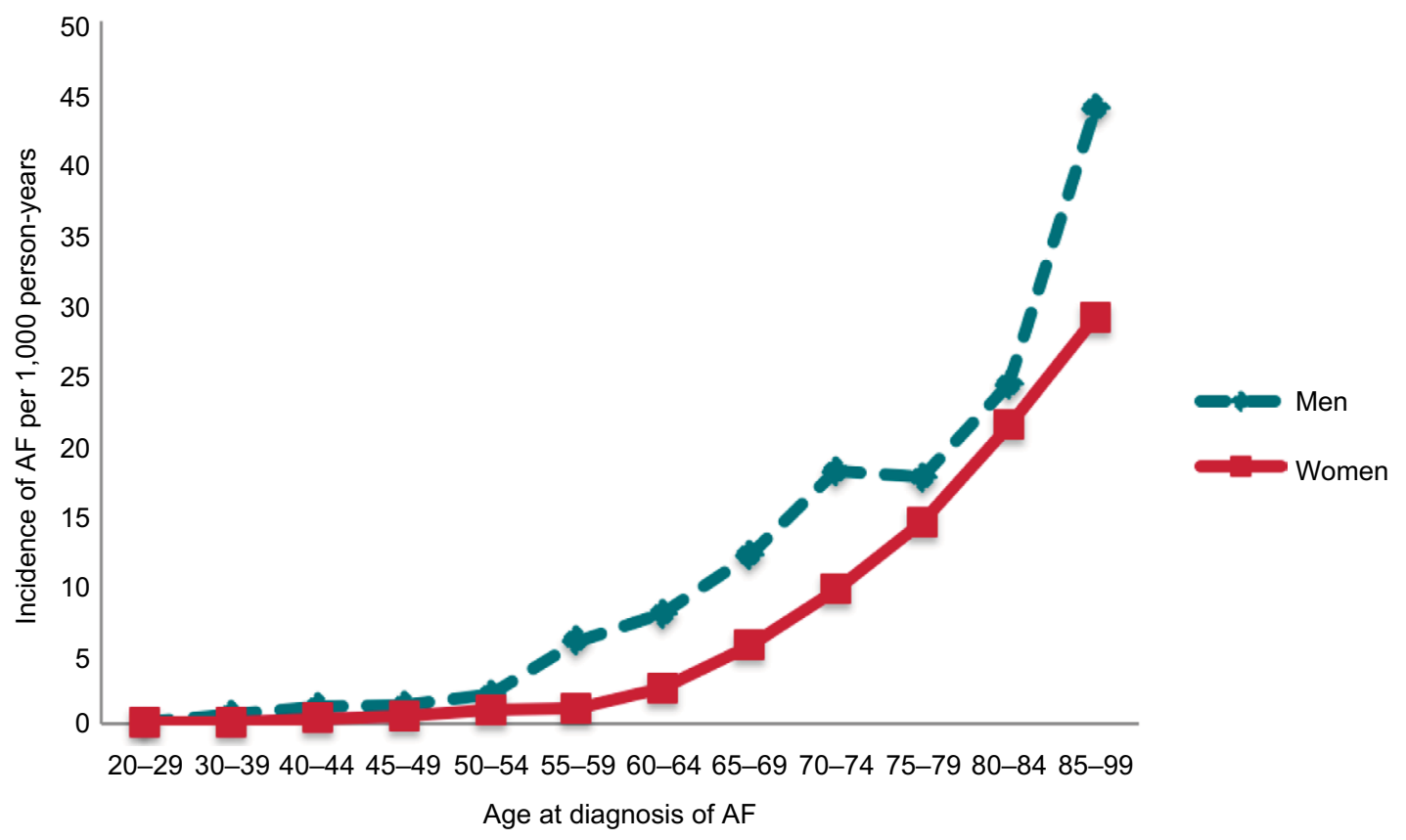

Figure 2 The incidence of first-diagnosed atrial fibrillation or atrial flutter (AF) according to age group and sex. 
Table 2 Characteristics of 607 patients with and without a provoking factor associated with first-diagnosed atrial fibrillation or atrial flutter (AF)

\begin{tabular}{lllllll}
\hline & $\begin{array}{l}\text { Cardiac } \\
\text { surgery } \\
\mathbf{n = 4 6}\end{array}$ & $\begin{array}{l}\text { Non-cardiac } \\
\text { surgery } \\
\mathbf{n = 2 1}\end{array}$ & $\begin{array}{l}\text { Acute } \\
\text { infection } \\
\mathbf{n = 2 4}\end{array}$ & $\begin{array}{l}\text { Myocardial } \\
\text { infarction } \\
\mathbf{n = 1 6}\end{array}$ & $\begin{array}{l}\text { Other }^{\mathbf{a}} \\
\mathbf{n = 1 8}\end{array}$ & $\begin{array}{l}\text { No provoking } \\
\text { factor } \\
\mathbf{n}=\mathbf{4 8 2}\end{array}$ \\
\hline $\begin{array}{l}\text { Age, years, mean } \pm \\
\text { standard deviation }\end{array}$ & $68.0 \pm 10.7$ & $73.6 \pm 10.8$ & $73.3 \pm 14.2$ & $78.3 \pm 8.5$ & $76.3 \pm 10.6$ & $73.8 \pm 12.3$ \\
$\begin{array}{l}\text { Female sex, } \mathrm{n}(\%) \\
\text { Deceased, } \mathrm{n}(\%)\end{array}$ & $12(26.1)$ & $\mathbf{8 ( 3 8 . 1 )}$ & $10(41.7)$ & $6(37.5)$ & $11(61.1)$ & $210(43.6)$ \\
\hline
\end{tabular}

Notes: a Other types of provoking factors included anemia (six patients), thyrotoxicosis or overdosage of levothyroxine (four patients), dehydration (two patients), acute abdominal pain (two patients), high tumor burden (two patients), aortic aneurysm (one patient) and status epilepticus (one patient). Data are reported as $\mathrm{n}$ (\%) or mean \pm standard deviation.

Table 3 Type of atrial fibrillation and atrial flutter (AF) in 607 patients with first-diagnosed AF

\begin{tabular}{|c|c|c|c|c|}
\hline \multicolumn{5}{|c|}{ Types of AF } \\
\hline & Single episode of AF, $n=147$ & Paroxysmal AF, $n=123$ & Persistent AF, $n=138$ & Permanent AF, $n=197$ \\
\hline $\begin{array}{l}\text { Age, years, mean } \pm \\
\text { standard deviation }\end{array}$ & $74.3 \pm 13.0$ & $74.4 \pm 9.5$ & $67.1 \pm 12.9$ & $78.4 \pm 10.1$ \\
\hline Female sex, n (\%) & $64(43.5)$ & $65(52.8)$ & $46(33.3)$ & $8 I(4 I . I)$ \\
\hline Provoking factor, $\mathrm{n}(\%)^{\mathrm{a}}$ & $45(30.6)$ & $23(18.7)$ & $37(26.8)$ & $22(11.2)$ \\
\hline $\begin{array}{l}\mathrm{CHA}_{2} \mathrm{DS}_{2}-\mathrm{VASc} \text {, mean } \\
\pm \text { standard deviation }\end{array}$ & $3.2 \pm 1.9$ & $3.4 \pm 1.7$ & $2.6 \pm 1.8$ & $3.7 \pm 1.8$ \\
\hline Deceased, n (\%) & $16(10.9)$ & $10(8.1)$ & $2(1.4)$ & $39(19.8)$ \\
\hline
\end{tabular}

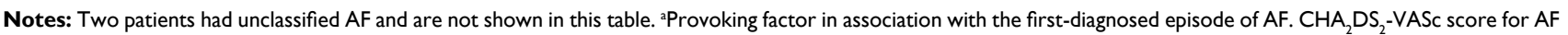
stroke risk: congestive heart failure, hypertension, age $\geq 75$ years (scored as 2 points), diabetes mellitus, previous stroke/TIA/arterial embolism (scored as 2 points), vascular disease, age $65-74$ years and female sex. Data are reported as $n(\%)$ or as mean \pm standard deviation.

\section{Types of AF}

The types of AF up to six months after the first-diagnosed episode of AF are shown in Table 3. Permanent AF was found in 197 (32.5\%), persistent $\mathrm{AF}$ in 138 (22.7\%), paroxysmal AF in $123(20.3 \%)$ and a single episode of AF in 147 patients (24.2\%). Patients with persistent AF were younger, more often men and had a lower mean $\mathrm{CHA}_{2} \mathrm{DS}_{2}$-VASc score compared to those with other types of AF.

\section{Mortality}

A total of 27 patients died within 30 days after diagnosis, and 41 died during the following five months. The cause of death within six months of the diagnosis of AF was cardiovascular disease in 38 patients (55.9\%), of whom 6 patients died of stroke. In addition, 14 patients $(20.6 \%)$ died of malignancy and 16 patients $(23.5 \%)$ from other causes. Cox proportional hazards regression with adjustment for age and sex was used to investigate the association between the presence of provoking factors for $\mathrm{AF}$ at diagnosis and the mortality risk. Compared to patients without a provoking factor, those with a provoking factor had an increased risk of death within 30 days (HR, 2.53; 95\% CI, 1.15-5.55) and within six months (HR, $1.77 ; 95 \% \mathrm{CI}, 1.04-3.01)$.

\section{Discussion}

This study showed that the incidence of AF was 4.0 per 1,000 person-years in an unselected Swedish population. There was a steep increase in the incidence of $\mathrm{AF}$ in the older age groups to 27.5 per 1,000 person-years in persons aged $\geq 80$ years. One in five patients had a provoking factor associated with the first episode of AF. Among patients with first-diagnosed $\mathrm{AF}$, one in four had no documented recurrence of AF during six months of follow-up. Four-fifths of the patients had a $\mathrm{CHA}_{2} \mathrm{DS}_{2}$-VASc score of 2 or more.

The incidence and age distribution of AF in our study were similar to those reported in German and Dutch studies. ${ }^{2,15}$ It is reasonable to assume that the incidence found in our study and the two above-mentioned studies, which used different methodologies and had different settings, can be used to estimate the number of new cases of $\mathrm{AF}$ among aging populations in other European countries.

We found that the ECG leading to the diagnosis of AF was registered due to suspected arrhythmia in barely onethird of the patients. A large proportion of patients were first diagnosed with $\mathrm{AF}$ when investigated for other heart diseases, predominantly ischemic heart disease. The proportion of truly asymptomatic patients with AF is difficult to estimate as symptoms of AF are diverse and include unspecific symptoms such as dizziness. Moreover, first-diagnosed AF sometimes occurs in severely ill patients, such as those with myocardial infarction or sepsis. In such cases, symptoms of the concomitant condition usually dominate the patient presentation. Furthermore, many conditions, for example, ischemic heart disease, where ECG registration is a part of the diagnostic 
workup, have a substantial symptom overlap with AF, and it is challenging to distinguish between symptoms caused by AF and by the other concomitant condition.

In the present study, the prevalence of provoking factors associated with the first-diagnosed episode of AF was $21 \%$ versus $31 \%$ in the Framingham study. ${ }^{5}$ In contrast to our study, the Framingham study excluded patients who died within 30 days of their first-diagnosed episode of AF and included data from an extended time period (between 1949 and 2012). Our study was performed between 2011 and 2012, and it thus reflects current healthcare practices in northern Sweden. Provoking factors, except for alcohol consumption, were defined in a similar way in both studies. Both the studies identified provoking factors in medical records; thus, the identification depends on the quality of the original data. Acute alcohol consumption was not registered in our study, which partly explains our lower rate of provoking factors. Altogether, this may have contributed to differences in the prevalence of provoking factors in the two studies.

The GARFIELD study is a recent study of patients with newly diagnosed AF. ${ }^{8}$ This prospective cohort study included patients with AF that was diagnosed within the past six weeks who had at least one risk factor for stroke. About $80 \%$ of the patients were enrolled by a cardiologist or internal medicine specialist. The age distribution was notably different between our study and that study. In the GARFIELD study, the mean age was 69.8 years, $31.3 \%$ were aged $<65$ years and $37.7 \%$ were aged $\geq 75$ years. In our study, the mean age was 73.5 years, $21.9 \%$ were aged $<65$ years and $48.4 \%$ were aged $\geq 75$ years. We speculate that elderly patients with newly detected AF were less often referred to a specialist clinic and were therefore not included in the GARFIELD study. The mean $\mathrm{CHA}_{2} \mathrm{DS}_{2}$-VASc score was similar in the two studies: 3.2 in the prospective GARFIELD cohort compared to 3.3 in our cohort. This may seem unexpected, since our population was older and would consequently be expected to have a higher mean $\mathrm{CHA}_{2} \mathrm{DS}_{2}$-VASc score. However, the higher age of our study population was balanced by the higher proportion of patients in our study who had a $\mathrm{CHA}_{2} \mathrm{DS}_{2}$-VASc score of 0 or $1(19.3 \%$ in our study compared to $16.5 \%$ in the GARFIELD study).

Permanent AF and persistent AF were more common in our study compared to the GARFIELD study. This may be explained by our use of a longer time period for the classification of AF (up to six months from the first diagnosis of AF compared to six weeks); this gave us more extensive data from the patient records and more data from the clinicians' assessments of the patients for classifying the type of
AF. In both studies, a patient who presents with AF for the first time is classified as a patient with "first-diagnosed AF", irrespective of the duration of the arrhythmia. ${ }^{8}$ During the follow-up time, patients were classified into other types of AF as more information about the patient's arrhythmia could be obtained and as AF is a progressive disease. Both studies used the classifications for paroxysmal, persistent and permanent AF. In our study, we further classified "first-diagnosed AF" as a "single episode of AF" if the first-diagnosed episode of AF was self-terminating and non-recurrent AF, that is, no additional documented episodes or clinical symptoms of AF for up to six months after the original diagnosis. A longer observational period is needed to better understand the extent to which patients will either progress to other types of AF or remain non-recurrent. When we compare the proportions of the types of AF between our study and the GARFIELD study, it is reasonable to compare the $24.2 \%$ of patients with a "single episode of AF" in our study with the $29.7 \%$ of patients with "new or unclassified AF" in the GARFIELD study.

In a previous cross-sectional study of patients with prevalent AF in northern Sweden, 50\% had permanent AF compared to $29 \%$ of incident cases in the present study. ${ }^{13}$ This is explained by the longer observational time in the study of prevalent cases and the progressive nature of the disease. In our study, $24 \%$ of patients had a single episode of AF. According to our definition, paroxysmal AF is recurrent while a single episode of $\mathrm{AF}$ is non-recurrent. The group with a single episode of AF had a higher prevalence of provoking factors and similar $\mathrm{CHA}_{2} \mathrm{DS}_{2}$-VASc scores compared to patients with paroxysmal AF. It is unclear whether patients with a single episode of AF, especially AF with a provoking factor, should be considered for rhythm control and anticoagulant treatment, as the long-term risk of recurrence is unknown. Unfortunately, current guidelines do not give any specific recommendations for this clinical situation.

The all-cause mortality was $11 \%$ during the first six months of our study. In a community-based study by Miyasaka et al, which was conducted between 1980 and 2000 , the mortality rate was $16.5 \%$ within the first four months. ${ }^{16}$ In our study, the most common causes of death were cardiovascular disease (54\%) and malignancy (24\%), which is comparable to the rates of $46 \%$ and $18 \%$, respectively, in the study by Miyasaka et al. ${ }^{16}$ The early mortality rate was high in both our study and Miyasaka et al's study, which suggested that AF represents a preterminal event in these cases. ${ }^{16}$ Consequently, severe illness may be the cause of AF as well as the cause of mortality. 
Our aim was to characterize a "real-life" population of patients with first-diagnosed AF. We therefore included all patients with a first-diagnosed episode of manually verified AF within the total population of a well-defined geographical area, regardless of patient age or type of AF. Furthermore, all previously identified 2,338 cases of prevalent $\mathrm{AF}$ were not at risk of developing $\mathrm{AF}$ and were thus excluded. This minimized the likelihood of including prevalent cases of AF in the incidence calculation, which is an inherent risk of all registry-based studies, and which can lead to an overestimation of the incidence. We previously showed that the diagnoses of AF registered in the NPR and in the diagnosis registers of primary healthcare providers are valid (96.5\% valid diagnoses), and that the registers are complete (incorporating $93.2 \%$ of all patients with AF). ${ }^{1}$ We also searched registries from both primary and secondary healthcare to include all patients with AF. One-fourth of the patients with AF included in our study were first diagnosed in primary healthcare and may therefore not have been identified if the search for AF cases only had been performed in hospital registries. In a previous Swedish study, $12 \%$ of all diagnoses of AF were found only in primary healthcare registries. ${ }^{17}$ Consequently, we think that our study population is representative of a reallife population of patients with $\mathrm{AF}$, and that our results are valid for other populations with a similar age structure. However, the majority of our participants were Caucasians, and there seems to be differences in AF disease burden in different ethnic groups. Therefore, it is uncertain whether our results can be generalized to populations with a broader representation of other ethnic groups. ${ }^{18}$

The major limitation of our study is the retrospective collection of patient data. The accuracy of our incidence rate and our evaluation of the type of AF depended on the patient having contacted a healthcare provider if they had symptoms of AF, as no study-specific patient interviews or ECG screenings were performed. Asymptomatic episodes of AF may have been missed, which would result in a lower incidence of $\mathrm{AF}$ and in overestimation of the proportion of single episode of AF. Information on risk factors for stroke and systemic embolism and presence of provoking factors for AF was also limited to what was documented in medical records. For example, it is known that alcohol use may provoke AF, but as we believe that data from medical records are an incomplete and often incorrect source of information on alcohol use, we chose not to register this factor. However, studies that are based solely on diagnostic codes from registries may underestimate the true prevalence of risk factors and provoking factors compared to our study, which was based on data from medical records.

\section{Conclusion}

In this Swedish population, the incidence of AF was 4.0 per 1,000 person-years, and one in five patients had a provoking factor at diagnosis of AF. Permanent AF was the most common type of AF. Four in five patients had a $\mathrm{CHA}_{2} \mathrm{DS}_{2}-\mathrm{VASc}$ score of 2 or more.

\section{Acknowledgments}

Financial support was provided by a regional agreement between Umeå University and the Västerbotten County Council for the cooperation in the fields of Medicine, Odontology and Health, and by the Society for Medical Research in Skellefteå.

\section{Disclosure}

The authors report no conflicts of interest in this work.

\section{References}

1. Norberg J, Bäckström S, Jansson JH, Johansson L. Estimating the prevalence of atrial fibrillation in a general population using validated electronic health data. Clin Epidemiol. 2013;5:475-481.

2. Wilke T, Groth A, Mueller S, et al. Incidence and prevalence of atrial fibrillation: an analysis based on 8.3 million patients. Europace. 2013;15(4):486-493.

3. Friberg L, Bergfeldt L. Atrial fibrillation prevalence revisited. J Intern Med. 2013;274(5):461-468.

4. Murphy NF, Simpson CR, Jhund PS, et al. A national survey of the prevalence, incidence, primary care burden and treatment of atrial fibrillation in Scotland. Heart. 2007;93(5):606-612.

5. Lubitz SA, Yin X, Rienstra M, et al. Long-term outcomes of secondary atrial fibrillation in the community: the Framingham Heart Study. Circulation. 2015;131(19):1648-1655.

6. Camm AJ, Lip GY, De Caterina R, et al; ESC Committee for Practice Guidelines-CPG; Document Reviewers. 2012 focused update of the ESC guidelines for the management of atrial fibrillation: an update of the 2010 ESC guidelines for the management of atrial fibrillationdeveloped with the special contribution of the European Heart Rhythm Association. Europace. 2012;14(10):1385-1413.

7. Camm AJ, Kirchhof P, Lip GY, et al; European Heart Rhythm Association; European Association for Cardio-Thoracic Surgery; ESC Committee for Practice Guidelines. Guidelines for the management of atrial fibrillation: the Task Force for the Management of Atrial Fibrillation of the European Society of Cardiology (ESC). Europace. 2010;12(10):1360-1420.

8. Kakkar AK, Mueller I, Bassand JP, et al; GARFIELD Registry Investigators. Risk profiles and antithrombotic treatment of patients newly diagnosed with atrial fibrillation at risk of stroke: perspectives from the international, observational, prospective GARFIELD registry. PLoS One. 2013;8(5):e63479.

9. Nabauer M, Gerth A, Limbourg T, et al. The Registry of the German Competence NETwork on Atrial Fibrillation: patient characteristics and initial management. Europace. 2009;11(4):423-434.

10. Lip GY, Laroche C, Dan GA, et al. A prospective survey in European Society of Cardiology member countries of atrial fibrillation management: baseline results of EURObservational Research Programme Atrial Fibrillation (EORP-AF) Pilot General Registry. Europace. 2014;16(3):308-319. 
11. Haim M, Hoshen M, Reges O, Rabi Y, Balicer R, Leibowitz M. Prospective national study of the prevalence, incidence, management and outcome of a large contemporary cohort of patients with incident non-valvular atrial fibrillation. J Am Heart Assoc. 2015;4(1): e001486.

12. Socialstyrelsen [homepage on the Internet]. Stockholm: The National Board of Health and Welfare. The National Patient Register. Available from http://www.socialstyrelsen.se/register/halsodataregister/patientregistret/inenglish. Accessed December 16, 2016.

13. Johansson C, Hägg L, Johansson L, Jansson JH. Characterization of patients with atrial fibrillation not treated with oral anticoagulants. Scand J Prim Health Care. 2014;32(4):226-231.

14. Halligan SC, Gersh BJ, Brown RD Jr, et al. The natural history of lone atrial flutter. Ann Intern Med. 2004;140(4):265-268.
15. Heeringa J, van der Kuip DA, Hofman A, et al. Prevalence, incidence and lifetime risk of atrial fibrillation: the Rotterdam study. Eur Heart J. 2006;27(8):949-953.

16. Miyasaka Y, Barnes ME, Bailey KR, et al. Mortality trends in patients diagnosed with first atrial fibrillation: a 21-year community-based study. J Am Coll Cardiol. 2007;49(9):986-992.

17. Forslund T, Wettermark B, Wändell P, von Euler M, Hasselström J, Hjemdahl P. Risk scoring and thromboprophylactic treatment of patients with atrial fibrillation with and without access to primary healthcare data: experience from the Stockholm health care system. Int J Cardiol. 2013;170(2):208-214.

18. Shen AY, Contreras R, Sobnosky S, et al. Racial/ethnic differences in the prevalence of atrial fibrillation among older adults - a cross-sectional study. J Natl Med Assoc. 2010;102(10):906-913. 


\section{Supplementary materials}

Table SI Detailed definitions of risk factors for stroke and systemic embolism and provoking factors for AF

Condition
Risk factors for stroke and systemic embolism:
Congestive heart failure

Hypertension

Diabetes mellitus

Previous stroke

Previous TIA

Previous arterial embolism

Vascular disease

Provoking factors for the first episode of AF:

Cardiac surgery

Non-cardiac surgery

Acute infection

Myocardial infarction

Anemia

Thyrotoxicosis or overdosage of levothyroxine

Dehydration

Acute abdominal pain

High tumor burden

Aortic aneurysm

Status epilepticus
Definition

ICD- I 0 diagnosis of congestive heart failure or LVEF $\leq 40 \%$ or at least moderately decreased left ventricular systolic function

ICD-I0 diagnosis of hypertension or receiving antihypertensive treatment

ICD-I0 diagnosis of diabetes mellitus or a diagnosis of diabetes mellitus in the medical record

ICD- 10 diagnosis of stroke or a diagnosis of stroke in the medical record

ICD-I 0 diagnosis of TIA or diagnosis of TIA in the medical record plus sudden onset of neurological symptoms and/or signs that lasted less than 24 hours

ICD-I 0 diagnosis of arterial embolism or a diagnosis of arterial embolism in the medical record

Previous myocardial infarction, objectively verified peripheral arterial disease (verified by appropriate imaging or treated with revascularization or amputation), complex aortic plaque or previous surgery for an arterial aneurysm

All types of cardiac surgery performed within 30 days preceding the first-diagnosed episode of AF (valvular repair or replacement surgery, coronary artery bypass grafting, percutaneous coronary intervention included)

All types of non-cardiac surgery performed within 30 days before the first-diagnosed episode of AF

A diagnosis of an acute infection in the medical record at the same time as the firstdiagnosed episode of AF

A diagnosis of a myocardial infarction in the medical record at the same time as the firstdiagnosed episode of AF

Anemia noted in the medical record at the same time as the first-diagnosed episode of AF

Thyrotoxicosis or overdosage of levothyroxine noted in the medical record as a provoking factor for the first episode of $A F$ at the same time as the first-diagnosed episode of AF

Dehydration noted in the medical record as a provoking factor for the first episode of AF at the same time as the first-diagnosed episode of $\mathrm{AF}$

Acute abdominal pain noted in the medical record as a provoking factor for the first episode of AF at the same time as the first-diagnosed episode of AF

High tumor burden noted in the medical record as a provoking factor for the first episode of $\mathrm{AF}$ at the same time as the first-diagnosed episode of $\mathrm{AF}$

Aortic aneurysm noted in the medical record as a provoking factor for the first episode of $\mathrm{AF}$ at the same time as the first-diagnosed episode of $\mathrm{AF}$

Status epilepticus noted in the medical record as a provoking factor for the first episode of $\mathrm{AF}$ at the same time as the first-diagnosed episode of $\mathrm{AF}$

Abbreviations: AF, atrial fibrillation or atrial flutter; ICD-10, the International Classification of Diseases-I0; LVEF, left-ventricular ejection fraction; TIA; transient ischemic attack. 
Table S2 Incidence of first-diagnosed atrial fibrillation and atrial flutter presented as number of incident cases per two years and incidence per I,000 person-years, by age and sex

\begin{tabular}{|c|c|c|c|c|c|c|}
\hline \multirow[t]{2}{*}{ Age, years } & \multicolumn{3}{|l|}{ Men } & \multicolumn{3}{|l|}{ Women } \\
\hline & Total population & Incident cases & Incidence & Total population & Incident cases & Incidence \\
\hline $0-19$ & 8,655 & 0 & 0 & 8,186 & 0 & 0 \\
\hline $20-29$ & 4,539 & I & 0.11 & 3,927 & 1 & 0.13 \\
\hline $30-39$ & 4,340 & 6 & 0.69 & 4,011 & 1 & 0.12 \\
\hline $40-44$ & 2,439 & 6 & 1.23 & 2,313 & 2 & 0.43 \\
\hline $45-49$ & 2,522 & 7 & 1.39 & 2,451 & 3 & 0.61 \\
\hline $50-54$ & 2,436 & 10 & 2.05 & 2,482 & 5 & 1.01 \\
\hline $55-59$ & 2,618 & 31 & 5.92 & 2,592 & 6 & 1.16 \\
\hline $60-64$ & 2,596 & 41 & 7.90 & 2,497 & 13 & 2.60 \\
\hline $65-69$ & 2,365 & 57 & 12.05 & 2,406 & 27 & 5.61 \\
\hline $70-74$ & 1,659 & 60 & 18.08 & $\mathrm{I}, 877$ & 36 & 9.59 \\
\hline $75-79$ & I, I89 & 42 & 17.66 & $\mathrm{I}, 598$ & 46 & 14.39 \\
\hline $80-84$ & 802 & 39 & 24.31 & 1,265 & 54 & 21.34 \\
\hline $85-89$ & 453 & 31 & 34.22 & 738 & 43 & 29.13 \\
\hline $90-94$ & 91 & 18 & 98.90 & 311 & 17 & 27.33 \\
\hline $95-99$ & 18 & 1 & 27.78 & 61 & 3 & 24.59 \\
\hline $100+$ & 0 & 0 & 0 & 4 & 0 & 0 \\
\hline
\end{tabular}

\section{Publish your work in this journal}

Clinical Epidemiology is an international, peer-reviewed, open access, online journal focusing on disease and drug epidemiology, identification of risk factors and screening procedures to develop optimal preventative initiatives and programs. Specific topics include: diagnosis, prognosis, treatment, screening, prevention, risk factor modification,

Submit your manuscript here: https://www.dovepress.com/clinical-epidemiology-journal systematic reviews, risk and safety of medical interventions, epidemiology and biostatistical methods, and evaluation of guidelines, translational medicine, health policies and economic evaluations. The manuscript management system is completely online and includes a very quick and fair peer-review system, which is all easy to use. 